\title{
Transformación institucional en democracia. El caso del voto facultativo para Fuerzas Armadas y Policía en Ecuador
}

\author{
Institutional Transformation in Democracy: \\ The case of the voluntary voting for police \\ and armed forces in Ecuador
}

\section{Diego Pérez Enríquez}

\section{Resumen}

Recepción: 10 de junio de 2013

Aceptación: 5 de agosto de 2013

Se analiza la incorporación del voto facultativo para las Fuerzas Armadas y la Policía Nacional en la Constitución de 2008 y sus efectos en la transformación institucional. Se hace un recuento desde el institucionalismo histórico a partir del retorno a la democracia (1979) de las condiciones bajo las que se relacionan la fuerza pública y los partidos políticos, con especial énfasis en la manera como tal estructura se acentúa a pesar de la Constitución de 1998. Además se evidencia el quiebre de estas relaciones a partir de la Constitución de 2008, donde se expande la base de ciudadanos habilitados para participar, y se plantea una aproximación contemporánea al ejercicio del voto por los miembros de la fuerza pública desde la perspectiva de la profundización de la democracia.

Palabras clave: Fuerzas Armadas, Policía Nacional, voto facultativo, institucionalismo histórico, Sistema Político, Ecuador.

\begin{abstract}
This article analyzes the incorporation of the right to vote of members of the armed forces and the police force in the 2008 Constitution and its effects on institutional transformation. From an historical institutionalism standpoint, it gives an account of the conditions under which law enforcement and the armed forces have related to political parties since Ecuador returned to democracy (1979), giving special emphasis to the way in which these relations deepened in spite of the Constitution of 1998. Furthermore, evidence is provided of the rupture of these relations with the Constitution of 2008, which increased the number of people with the right to vote in the country. The article also proposes a contemporary approach to the exercise of the right to vote of those in the armed forces and the police force from the perspective that it deepens democracy.
\end{abstract}

Keywords : Armed forces, Ecuadorian police force, voluntary voting, historical institutionalism, political system, Ecuador. 


\section{Introducción}

ste artículo se introduce en la transformación institucional como consecuencia de la posibilidad de que los miembros de la fuerza pública hayan adquirido derecho facultativo al voto con la Constitución de 2008. Tomando la aproximación teórica derivada del institucionalismo histórico, este artículo parte de un esfuerzo por ubicar los cimientos sobre los que se ha construido el sistema político, tomando como punto de partida el retorno a la democracia en 1979 . Dado que, a partir de tal ańo, se establecen buena parte de las condiciones bajo las que se relacionan la fuerza pública y los partidos políticos, se hace particular énfasis en la manera como tal estructura se acentúa a pesar de la Constitución de 1998. El punto de quiebre llega en 2008, donde se introducen una serie de dispositivos tendientes a fortalecer la institucionalidad democrática del país en términos de la expansión de la base de ciudadanos habilitados para participar en las elecciones.

Las siguientes páginas están divididas en dos secciones. La primera parte analiza brevemente los detalles del sistema político conformado tras la transición tomando como actores a las instituciones de la fuerza pública y a los partidos políticos. La segunda sección plantea una aproximación contemporánea al ejercicio del voto por los miembros de la fuerza pública desde la perspectiva de la profundización de la democracia.

Si bien el centro de preocupación es la fuerza pública como unidad, se debe reconocer que este documento enfatiza en el rol de Fuerzas Armadas (FFAA), principalmente por la línea de investigación personal realizada, pero también porque en el periodo señalado la Policía Nacional mantiene un rol secundario en la dimensión política que se trabaja aquí. Con la reflexión que se aprieta en estas pocas páginas se busca incentivar miradas hacia ámbitos diversos de comprensión de la naturaleza del sistema político ecuatoriano y los momentos de ruptura que han implicado transformaciones en su trayectoria histórica. 


\section{Una breve aproximación al sistema político postransición}

Una reflexión sobre el voto facultativo para los miembros de la fuerza pública debe comenzar, inevitablemente, de aproximarse a la manera cómo se ha estructurado el sistema político en el Ecuador. Sucintamente, tras la noción de sistema político se plantea la intención de "interpretar la vida política como una serie compleja de procesos mediante los cuales ciertos tipos de inputs se convierten en el tipo de outputs que podemos denominar políticas autoritarias, decisiones y acciones ejecutivas..." (Easton, 2001: 221), lo cual supone aproximarse al problema planteado desde la comprensión de sus actores como parte de un sistema de conducta que inevitablemente está influido por aquello que ocurre en su entorno. Tal como plantea el mismo autor, su análisis no se verifica en el vacío, sino que se lo debe comprender en la complejidad y vastedad de interacciones que lo atraviesan. Desde esta perspectiva se vuelve inevitable trazar el ámbito de análisis del sistema, previo a discernir sus operaciones.

Temporalmente, se parte en el análisis con el momento fundacional que se da con la Constitución de 1978 y la transición desde un régimen dictatorial a uno democrático. Se establece una comparación entre tal momento con el que desencadena la Constitución de 2008, cuyo análisis se trabaja en la siguiente sección. Por la naturaleza de la transición, es pertinente considerar a los dos actores que intervienen en su negociación (inputs), así como evaluar las operaciones (outputs) a las que dan origen. El reducido espacio del artículo impide profundizar en varios de los hechos que aquí únicamente se mencionan.

El sistema político ecuatoriano, tras la transición de 1979, se dividió en dos niveles de funcionamiento prácticamente impermeables entre sí: las instituciones de la fuerza pública, por un lado, y los partidos políticos, por otro. En el caso de las primeras es pertinente una distinción adicional entre FFAA y Policía Nacional, donde las primeras operaron dentro de una lógica estrechamente vinculada con la tradición de retornos a la democracia mediante pactos con los partidos políticos; y la segunda, como se ve más adelante, operó de manera que su visibilidad 
política fue menor, lo que no implica que haya sido menos activa. En el otro lado de esta ecuación, los partidos políticos coparon la administración de la democracia, subsumiendo una variedad de temas que se extendían desde la representación hasta el acceso y mantenimiento de la burocracia. Las siguientes líneas buscan dar cuenta de cómo la estructura creada en el momento de la transición a la democracia nace una suerte de marca de nacimiento que influye sobre la manera en que se lleva a cabo la participación política en el país hasta la promulgación de la Constitución de 2008. El enfoque inicial está con las actuaciones de las FFAA y de los partidos políticos, para finalmente introducir una reflexión sobre la Policía Nacional.

Las FFAA ecuatorianas -al igual que sus pares latinoamericanos- aseguraron su nueva posición en la democracia a través de “... derechos o privilegios adquiridos de manera formal o informal para ejercer un control efectivo sobre su institución sin interferencia de los civiles" (Olano, 2001: 45). El establecimiento de estos espacios clausurados y privilegiados, denominados como prerrogativas militares ${ }^{1}$ (Stepan, 1988) garantizó condiciones favorables para la intervención castrense en política bajo la figura del "retiro de apoyo" al Presidente (1997, 2000 y 2005), aunque también generó incentivos para intentos de golpe de Estado (2000). De cualquier manera, la perspectiva militar de frente al retorno a la democracia se estructuró bajo la noción de la profesionalización y el distanciamiento institucional de la cotidianidad del ejercicio del poder. $\mathrm{Al}$ menos en teoría. El hecho es que, tal como describe Loveman (1999), en el caso ecuatoriano se creó un sentido de "protección de la democracia" que surgió de manera simultánea con el Estado.

1 Estas incluyen: 1) Sistema de ascensos autorregulado; 2) definición propia de los sistemas y contenidos de la educación castrense; 3) estrategias establecidas por la propia institución en ausencia de la opinión e intervención civil; 4) un rol independiente en el sistema político por parte de los militares; 5) posición de asesores y consejeros presidenciales exclusivos en cuestiones de seguridad y defensa; 6) autonomía y control de los servicios de inteligencia; 7) un papel activo en la administración de empresas del Estado, sea a través de posiciones en sus directorios o en el manejo ejecutivo de las mismas; 8) mantenimiento de un fuero privativo sobre sus acciones, y, en el caso de Ecuador, estas incluirían también la garantía de amplios espacios de acción para misiones de desarrollo interno. 
Bajo el sentido de la democracia protegida, se parte de la noción de que existe un foco de autoridad residual en una institución del Estado para este caso las FFAA, aunque en otros pueden ser partidos políticos, la Iglesia, etc.- con independencia de quien sea el detentador formal del poder. Desde ese espacio de autoridad residual se procura influir en la implementación del proyecto de Estado, incorporando sentidos político ideológicos afines a una concepción particular de cómo este debería operar. Así, desde tal posición se influencian las estructuras institucionales del Estado, introduciendo, además, dispositivos que permiten la intervención legal (y hasta legítima) cuando el proyecto se desvía de aquello que se considera aceptable. En el planteamiento de Loveman, los ejemplos más patentes de tales dispositivos se ubican las constituciones latinoamericanas desde la fundación misma de los Estados, donde se establecía como misión de las FFAA la garantía del orden institucional, la defensa de la soberanía y la protección de los intereses nacionales y el bien común (1994). La ambigüedad tras tales advocaciones e interpretaciones viabilizó una serie de intervenciones a lo largo de la región.

En el caso ecuatoriano, sin diferir de lo descrito, se debe considerar la existencia de un freno que limitaba cíclicamente cualquier incentivo de intervención y permanencia prolongada en el costado político de la administración del Estado. La protección de la soberanía territorial era un objetivo primordial debido a la permanente tensión fronteriza con Perú y en 1941, tras la derrota, se profundizaron los procesos de profesionalización militar. Buena parte de las decisiones, a partir de entonces, procuraron desvincular a esta institución del modelo de control civil subjetivo (Huntington, 1995) que había primado para operar en una lógica en que las definiciones de prioridades y acciones sugieran de la misma institución. En parte, el proceso de transición se puede explicar por la preocupación que generaba, en las filas militares, el alejamiento de la misiones profesionales por estar involucrados en la administración del Estado. Así, al momento de la transición, las FFAA ecuatorianas procuraron estructurar un sistema que les permita mantener la protección del sistema sin afectar la profesionalidad en el cumplimiento 
de su misión fundamental (Bustamante, 1988), de allí que la opción de involucramiento en política fuera mediante intermitentes llamados de atención y advertencias sobre las formas de la administración del poder, y episodios de manejo del poder limitados muy severamente en el tiempo y el espectro del mismo (Bustamante, 1998; Fitch, 1998).

No se puede dar cuenta, sin embargo, de la dinámica de funcionamiento del sistema político ecuatoriano si es que no se toma en consideración la manera cómo se articularon los partidos políticos, aquel otro costado de la transición que se tiende a pasar por alto, para dar viabilidad al retorno a la democracia. Brevemente se debe destacar que el diseńo institucional de la transición, desde la perspectiva partidista, se enfocó en la preservación de espacios exclusivos y excluyentes de la intervención ciudadana ${ }^{2}$, en el manejo burocrático-institucional.

Retomando la noción de las prerrogativas en el sentido planteado previamente, en este caso se pueden observar una serie de prerrogativas partidistas, espacios que los partidos delimitaron en el contexto de la transición para su exclusivo control: la regulación del acceso de nuevos actores políticos a través de la creación y control partidista del organismo electoral ${ }^{3}$; la capacidad de limitar constitucionalmente cualquier iniciativa a través de la creación de un tribunal con exclusividad en temas constitucionales y operación independiente del poder judicial; y el reparto del poder decisorio entre Ejecutivo y Legislativo para la elección de magistrados a la Corte Suprema de Justicia.

2 Es importante diferenciar el concepto de ciudadanía del más genérico "civiles". En tal sentido, se entiende al primero como la unidad mínima del Estado, detentador de derechos y obligaciones, que se reconoce como tal y por lo tanto opera en una lógica de exigencia de los mismos, mientras que el segundo refiere únicamente a quienes no son parte de la institución militar, sin entrar en la consideración de su condición política según los términos antes planteados.

3 En esta operación se proveyeron mecanismos de sanción y de incentivo, los primeros atados principalmente al cumplimiento de los límites de gasto electoral; los segundos vinculados a la provisión de fondos cuyo monto variaba en relación al desempeńo electoral. La administración ad hoc de sanciones y la aplicación electiva de las normas relativas a los umbrales electorales para la permanencia de los partidos, en función de los pactos legislativos predominantes, demuestran la dinámica de operación del TSE entre 1979 y 2006. 
En este contexto, el eje de funcionamiento planteado por los partidos políticos para administrar la democracia, tras la transición, giró en torno a reivindicarse como el intermediario óptimo en la relación ciudadanía-gobierno-Estado. Los dispositivos constitucionales y legales construidos -sobre los que no se abunda aquí- permitieron referenciar cualquier decisión a la figura del partido, haciendo que la operación de las instituciones vinculadas a los temas electorales y a los constitucionales dependa de la configuración predominante de fuerzas políticas. La función judicial, por otra parte, dependía directamente del costado del que cedieran las tensiones Ejecutivo-Legislativo.

Las mencionadas prerrogativas políticas destacan un primer punto primordial para este análisis: las agrupaciones políticas que dirigieron la transición concebían a los partidos como instituciones que "organizan la caótica voluntad pública” (Sartori, 2000: 57), motivo por el que se les asignó un carácter nuclear en la estructura del Estado. Desde esta concepción, los partidos -y solo ellos- poseían el conocimiento y la expertise necesaria para lograr que el Estado respondiera de manera eficiente sobre las quejas y solicitudes de los ciudadanos. Se partía del supuesto que la organización partidaria financiada por el Estado invertiría tales recursos en profesionalizar sus cuadros, fomentar la participación masiva de la ciudadanía y lograr una organización interna desarrollada y democrática que le garantizara un nivel aceptable de supervigilancia sobre sus miembros activamente vinculados a la administración pública. El acceso democrático a cargos públicos supondría el nivel mayor de realización para sus miembros, pues tendrían la posibilidad de utilizar los recursos del Estado para responder a las necesidades de sus electores, según el lineamiento ideológico con el que comulgaban. Toda esta imagen se concluía con la existencia de una mano invisible del mercado electoral, según la cual la responsabilidad del partido y de sus miembros en cargos públicos estaba atada indisolublemente a las probabilidades de éxito electoral. Así, se presumió que a mayor eficiencia y honradez, mayor número elecciones ganadas.

Esta preconcepción, no obstante, erró al no reconocer elementos de una estructura más compleja en la operación del Estado, lo que generó 
bloqueos atrás de los cuales la única iniciativa de solución, encabezada desde los mismos partidos, pasaba por los "intentos de reformar el sistema político del país, cambiando regulaciones electorales e incluso la Constitución [y] tentativas de establecer [en muchos casos restablecer] vínculos clientelares directos entre la sociedad y el gobierno. Ambas respuestas pasarían a ser verdaderas reglas de juego permanentes en los ańos 1996-2006" (Andrade, 2009a: 54-55).

Bajo tal contexto, la relación partidos políticos-FFAA no existió básicamente, salvo en aquellos momentos en que se requería de la legitimación castrense (real o simbólica) que permitiera resolver las tensiones coyunturales, tal como había ocurrido en los conflictos políticos precedentes a la transición (Andrade, 2009b; Varas, 1991; Fitch, 1986). Por fuera de tales circunstancias, esta relación permaneció restringida a temas puntuales, como ocurrió durante la guerra del Cenepa, en $1995^{4}$.

El rol de la Policía Nacional en la configuración del sistema político postransición ha sido menos claro. Quizás se puede interpretar su situación desde el proceso de construcción de capacidades internas y de su propia definición institucional. Parecería claro que tal ejercicio remitió a la Policía a una serie de esfuerzos por a) diferenciarse de las FFAA justificando su existencia a través de una búsqueda de autonomía y diversificación en su ámbito de acciones y, b) servir muy estrechamente al poder político de turno desde una posición absolutamente obediente. El hecho es que tales limitaciones restringieron la construcción de una política de seguridad ciudadana, planteando el horizonte de acción de la institución básicamente en la lógica todavía referenciada en la doctrina de la seguridad nacional (Pontón, 2008; Hanashiro \& Pontón, 2007). La posición que adopta la institución tras la transición se restringió a los complejos procesos de adaptación a una normativa y acciones vinculadas con el respeto de derechos humanos, la generación de capacidades de respuesta institucional ante las demandas internacionales para que se involucre en misiones de control antinarcótico, y un prolongado proceso -aún no

4 Se debe destacar que recién bajo el orden constitucional vigente desde 2008 la Asamblea Nacional conforma una comisión especializada, la de Soberanía, Integración, Relaciones Internacionales y Seguridad Integral, ocupada, entre otros, de aquello que hace al funcionamiento de las FFAA. 
concluido- de redefinición y modernización que debería ponerla a tono con demás procesos en marcha en la región (Frühling, 2009).

De lo señalado se puede advertir una dicotomía en los intereses que han primado sobre el sistema político por parte de los actores militares y los actores partidistas. Ésta, en última instancia, ha llevado a que dos tipos de agenda autorreferenciada generen la operación de estos actores desde una distancia que no fue saldada ni siquiera a través de iniciativas que explícitamente procuraban tal acercamiento (por ejemplo, la construcción del Libro Blanco de la Defensa Nacional en sus dos ediciones ${ }^{5}$ ). En tal dinámica de operación, el ejercicio del voto se consideró uno de los beneficios a los que accederían únicamente aquellos ciudadanos que estaban bajo el paraguas de las prerrogativas partidistas (la mayoría de la población), en tanto que los miembros de la fuerza pública, por su naturaleza apolítica (asignada por el sistema, pero también asumida por ellos), no intervendrían del acto electoral.

\section{Cuestiones sobre el voto: hacia una democracia de ciudadanos y ciudadanas}

Partiendo de las nociones minimalistas de democracia (Munck, 2005; Weyland, 2004; Mainwaring, Brinks, \& Pérez-Liñán, 2001; Schumpeter, 1986) hacia aquellas más complejas (Touraine, 2001; Lefort, 1988; Lipset, 1959), un breve recorrido sobre la teoría de la democracia permite observar que una de sus piedras angulares es la participación de los ciudadanos en procesos que permitan la elección de individuos que actúen en su nombre conformando un gobierno. Las maneras en que este se conforma varían tanto como sus participantes. El centro de las discusiones clásicas en teoría política se interrogan sobre quién gobierna, cómo llega allí y de qué manera permanece en tal posición ${ }^{6}$; pero este documento no se plantea tales interrogantes.

5 El Libro Blanco de la Defensa se publicó por primera vez en 2002, y posteriormente se realizó una actualización del mismo en el año 2006.

$6 \mathrm{Al}$ respecto se puede revisar un breve pero acertado compendio de Norberto Bobbio. 2001. La teoría de las formas de gobierno en la historia del pensamiento político. Fondo de Cultura Económica, México. 
Basta decir que, en la misma transición el Ecuador, en el Artículo 1 de la Constitución de 1978 se define como un Estado cuyo "gobierno es republicano, electivo, responsable y alternativo..." (Constitución Política del Ecuador, 1978). Desde la perspectiva republicana, este enunciado implica la adopción de un criterio de libertad como no dominio ${ }^{7}$, en términos de la responsabilidad estatal, para garantizar las mismas libertades y aplicabilidad de la ley de manera equitativa para todos sus ciudadanos, además de la división del poder entre varias funciones con el fin de limitar las posibilidades de que el gobierno tome decisiones arbitrarias (Pettit, 1997). Hacerlo electivo, responsable y alternativo, por otra parte, traslada el centro decisorio a los ciudadanos a través de una capacidad para votar periódicamente y, en algunos casos, de ser electos.

En la estructura del sistema político que se esbozó en líneas previas, se planteaba la distancia entre las instituciones de la fuerza pública y los partidos políticos, que habían tomado para sí todo el espectro de posibilidades de participación ${ }^{8}$, mientras que las primeras, en general, habían tendido a ocuparse de sus procesos profesionales. Este acuerdo se garantizó constitucionalmente mediante el Artículo 33, con el que se excluía la posibilidad de que los miembros de la fuerza pública ejercieran el derecho al voto, al tiempo que el Artículo129 reiteraba la condición no deliberante que estas tenían. Tales disposiciones se pueden interpretar como una reacción directamente vinculada a la culminación del periodo de dictadura y la intención de no generar incentivos para un potencial retorno militar a la política. Por otra parte, el compromiso transicional de mantenerse dentro de los cuarteles implicaba este tipo de cesiones.

Más allá de las razones prácticas atrás de la decisión, el hecho es que el sistema político de 1979 se estructuró de manera que contradecía su

7 En honor al espacio se reduce el argumento en torno a las concepciones de libertad como no dominio: todos operan desde posiciones iguales, y de libertad como no interferencia: nada podría interferir con las decisiones de un individuo, y de todos modos sus posibilidades de acción estarían limitadas una condición de subsumisión ante otro individuo.

8 El Artículo 37 de la Constitución restringía las posibilidades de participación como candidato únicamente a los miembros afiliados a un partido. 
propia naturaleza republicana, creando desigualdades intrínsecas entre sus ciudadanos - no todos tenían las mismas libertades- y limitando la democracia en la variable de la participación. Tal estructura se mantuvo en la Constitución de 1998.

Existen regularidades históricas que influyen sobre la trayectoria que siguen los sistemas políticos y permiten presuponer resultados más o menos equiparables en el tiempo, pero ello no descarta la posibilidad de que las instituciones cambien, particularmente en coyunturas criticas que desencadenan transformaciones (Mahoney \& Thelen, 2010; Immergut, 2005; Thelen, 1999). El proceso constituyente de 2007-2008 se puede considerar una de tales coyunturas en las que se introdujo una reforma importante en términos de "democratización de la democracia" al ampliar la base de ciudadanos en capacidad de ejercer su derecho al voto.

Si bien la naturaleza de los miembros de la fuerza pública es particular al considerar su condición como parte de la única institución que detenta el monopolio legítimo de la fuerza, esta función no debería implicar una restricción en sus derechos ciudadanos. Ampliar la base de participantes conlleva la extensión de la legitimidad que ampara las decisiones de un gobierno; en tal contexto es pertinente "descubrir y entender que la disidencia, a diversidad de opiniones, el contraste, no son enemigos de un orden político-social" (Sartori, 1993: 148), restando la responsabilidad de lograr que tal pluralismo genere cohesión en el funcionamiento del Estado. Al considerar que existía un discurso predominante que planteaba las preocupaciones por la participación de la fuerza pública como electores en la incertidumbre porque ésta se convirtiera en un grupo politizado, con capacidad de acción que, además, superaba la de cualquier otro actor en la sociedad, respondía a un momento histórico institucional distinto. Retomando el principio de interpretación del institucionalismo histórico, existía una dependencia de trayectoria atada a una institución (Immergut, 2005) que operaba como el sumo decisor en momentos de tensión política. Bajo esa óptica, un miembro que respaldara abiertamente una opción y decidiera por ella constituía un riesgo. 
La importancia del Artículo 62 en la Constitución de 2008 radica justamente en la expansión de la democracia incorporando una dimensión de ciudadanía a individuos que, por una opción profesional, habían sido largamente excluidos de la posibilidad de expresarse sobre un gobierno que de todos modos tomaba decisiones sobre ellos. Haciéndolo facultativo y reglamentando posteriormente las condiciones para realizar proselitismo en los recintos militares, se genera un límite importante que aclara las condiciones de involucramiento, pero quizás la más clara y eficiente manera de contener cualquier aventura política de la fuerza pública radica en el fortalecimiento institucional y profesionalización de FFAA y la Policía Nacional. Si bien el camino por hacer aún es largo, es importante notar que las consecuencias para la democracia son a priori positivas al extender el número de ciudadanos que participan de las decisiones que toma el Estado.

\section{Conclusiones}

En las páginas precedentes se han resaltado elementos relativos al ejercicio del voto considerado desde la perspectiva del institucionalismo histórico. Con el fin de concluir el presente se debe considerar, primeramente, que el centro del problema tras las intervenciones militares -sin descontar las de la Policía - no radica en su ejercicio del voto. Al mirar la estructura del sistema político postransición se encuentra que tal derecho no se otorgó a los miembros de tales instituciones y, sin embargo, ello no restringió la posibilidad de intervención desde las mismas.

Justamente, en el sentido de las restricciones y desde una perspectiva más centrada en los principios de la democracia, garantizar el voto con algún tipo de discriminación implica la creación de ciudadanos de categorías distintas, pues no todos pueden marcar su preferencia sobre quiénes tomarán decisiones por ellos. Así, la ampliación de derechos amplia la libertad en el funcionamiento de la democracia puesto que se constituye un número mayor de ciudadanos de plenos derechos que, además, operan con menos restricciones sobre sí. 
La preocupación por el control civil objetivo (Huntington, 1995) que se ejerza sobre la fuerza pública y la democratización de la misma no se logran mediante la restricción de derechos, sino a través del establecimiento minucioso de funciones y responsabilidades en las instituciones del Estado. Considerar a los miembros de la fuerza pública como ciudadanos de plenos derechos únicamente puede fortalecer a sus instituciones, en tanto en su interior el proselitismo se restrinja y, por otra parte, no se presione sobre sus miembros para que tomen una determinada preferencia. Es central afrontar los retos de la institucionalización de la democracia para profundizarla.

\section{Bibliografía}

Andrade, P. (2009a). La era neoliberal y el proyecto republicano. Quito: Universidad Andina Simón Bolivar-Corporación Editora Nacional. (2009b). Democracia y cambio politico en el Ecuador: Liberalismo, politica de la cultura y reforma institucional. Quito: Universidad Andina Simón Bolívar-Corporación Editora Nacional.

Bustamante, F. (1988). "Los militares y la creación de un nuevo orden democrático en Perú y Ecuador”. Documento de Trabajo (370). Santiago: FLACSO.

Bustamante, F. (1998). "Democracy, Civilizational Change and the Latin American Military”. En Fault Lines of Democracy in Post - Transition Latin America. F. Agüero \& J. Stark (ed.). Miami: North South Center Press at the University of Miami.

Easton, D. (2001). Categorías para el análisis sistémico de la política. En Diez textos básicos de ciencia politica. A. Batlle (ed.): 221-230. Barcelona: Ariel.

Fitch, S. (1998). The Armed Forces and Democracy in Latin America. Baltimore: The John's Hopkins University Press.

(1986). "The military Coup d'État as a political process: a general framework and the Ecuadorian case". En Armies and Politics 
in Latin America. A. Lowenthal \& S. Fitch (ed.). New York: Holmes $\&$ Meier.

Frühling, H. (2009). Violencia y policía en América Latina. Quito: FLACSO.

Hanashiro, O., \& D. Pontón (2007). "Elementos para una reforma policial en el Ecuador". En Ciudad Segura. Debates sobre seguridad ciudadana. A. Santillán, J. Ponton \& D. Ponton (ed.): 123-140. Quito: FLACSO. Huntington, S. (1995). El Soldado y el Estado. Buenos Aires: Grupo Editor Latinoamericano.

Immergut, E. M. (2005). "Historical-Institutionalism in Political Science and the Problem of Change". En Understanding Change. Models, Methodologies and Metaphors. A. Wimmer, \& R. Kössler (ed.): 237-259. Houndmills, England: Palgrave Macmillan.

Lefort, C. (1988). Democracy and political theory. Cambridge: Polity Press. Lipset, S. M. (1959). "Some social requisites of democracy: economic development and legitimacy". En The American Political Science Review. 53 [1]: 69-105.

Loveman, B. (1999). For la Patria. Politics and armed forces in Latin America. Wilmington: Scholarly Resources.

Mahoney, J., \& Thelen, K. (2010). "A theory of gradual institutional change". En Explaining institutional change. Ambiguity, agency and power. J. Mahoney \& K. Thelen (ed.): 1-37. New York: Cambridge University Press.

Mainwaring, S., Brinks, D., \& Pérez-Liñán, A. (2001). "Classifying Political Regimes in Latin America, 1945-1999”. En Studies in Comparative International Development. 36 [1]: 37-65.

Munck, G. (2005, Marzo 15). Democracy and institutions in Latin America: reforms without illusions (Visita del 23 de junio en Política Comparada: http://www.politicacomparada.com.ar/material/ articulos/Democracia-y-sistemas-Politicos/2-Democracy-and-Institutions-in-Latin-America-Gerardo-L-Munck.pdf

Olano, A. (2001). Las relaciones cívico militares y la caida de la democracia en el Perú. European Press Academic Publishing. 
Pettit, P. (1997). Republicanism. A theory of freedom and government. Oxford: Oxford University Press.

Pontón, D. (2008). La reforma policial en el Ecuador: un tema relegado al olvido. En Seguridad multidimensional en América Latina. F. Rivera (ed.): 451-482. Quito: FLACSO.

Sartori, G. (2000). Partidos y Sistemas de partidos. Madrid: Alianza. (1993). ¿Qué es la democracia? México D.F: Alianza.

Schumpeter, J. (1986). "Democracy as competition for leadership". En Political Leadership. A sourcebook. B. Kellerman (ed.): 199-203. Pittsburgh: University of Pittsburgh Press.

Stepan, A. (1988). Rethinking Military Politics: Brazil and the Southern Cone. Princeton: Princeton University Press.

Thelen, K. (1999). "Historical institutionalism in comparative politics”. En Annual Review of Political Science. 2: 369-404.

Touraine, A. (2001). ¿Qué es la democracia? México D.F: Fondo de Cultura Económica.

Varas, A. (1991). "Las Relaciones Civil - Militares en la Democracia”. En América Latina, Militares y Sociedad. D. Kruijt, \& E. Torres-Rivas (ed.). San José: FLACSO.

Weyland, K. (2004). "Neoliberalism and Democracy in Latin America: A Mixed Record”. En Latin American Politics \& Society. 46 [1]: 135-157.

\section{Normativa}

Constitución de la República del Ecuador (1978). Congreso Nacional. Quito (codificada en 1984). R.O. N. ${ }^{\circ} 59$.

Constitución de la República del Ecuador (1998). Asamblea Nacional Constituyente. Quito. Decreto Legislativo N. 000.

Constitución de la República de Ecuador (2008). Asamblea Constituyente. Ciudad Alfaro.

Ministerio de Defensa Nacional del Ecuador (2002). Libro Blanco de la Defensa Nacional. Quito. 